\title{
On the estimation of reflectivity in reverse-time migration
}

Natiê Albano*, Jessé Costa (UFPA \& INCT-GP), Jörg Schleicher, and Amélia Novais (UNICAMP \& INCT-GP)

Copyright 2021, SBGf - Sociedade Brasileira de Geofísica

This paper was prepared for presentation during the $17^{\text {th }}$ International Congress of the Geophysical Society held in Rio de Janeiro, Brazil, 08 to 11 November 2021.

Contents of this paper were reviewed by the Technical Committee of the $17^{\text {th }}$ International Congress of the Brazilian Geophysical Societyldeas and concepts of the text are authors' responsibility and do not necessarily represent any position of the SBGf, its officers or members. Electronic reproduction or storage of any part of this paper for commercial purposes without the written consent of the Brazilian Geophysical Society is prohibited.

\section{Abstract}

The inverse scattering imaging condition (ISIC) for reverse-time migration (RTM) is based on a combination of temporal and spatial derivatives of the up- and downgoing wavefields. In the highfrequence regime, this image condition is exactly the inverse of Born modeling, which justifies it being called a true-amplitude imaging condition. By means of partial integrations (or redistribution of the frequency factors in the frequency domain), we derive several alternative expressions for this imaging condition. Interestingly, the temporal derivatives can be completely replaced by spatial derivatives and temporal integrations. One version of the ISIC makes use of the Laplacian operator, in this way relating to a common way of removing backscattering artifacts in RTM. We demonstrate by numerical examples using the Marmousi II model that the theoretical equivalence of the equations leads to similar, but not identical, images. Our numerical tests indicate that the ISIC based on the spatial derivatives are the most economic ones, and that the images obtained using the secondtime derivative of the source wavefield show a slightly improved resolution over the other implementations.

\section{Introduction}

Reverse-time migration (RTM) is a seismic imaging method based on the full (two-way) wave equation (Schultz and Sherwood, 1980; McMechan, 1983; Baysal et al., 1983) to propagated the involved wavefields into the subsurface. In the same way as other wave-equation based migration techniques, it makes use of an image condition, the most basic form of which is a simple cross-correlation of the forward-propagated source wavefield $\left(p_{s}\left(t, \mathbf{x} ; \mathbf{x}_{s}\right)\right)$ and the backward-propagated receiver wavefield $\left(p_{r}\left(t, \mathbf{x} ; \mathbf{x}_{r}\right)\right)$ (Claerbout, 1971). Frequently, a Laplacian filter is applied to the migrated image to attenuate backscattering noise, and an illumination factor $\left(P\left(\mathbf{x} ; \mathbf{x}_{s}\right)\right)$ is used to compensate for the loss of amplitude with increasing depth (Biondi, 2006), i.e.,

$$
I_{c}(\mathbf{x})=\frac{1}{P\left(\mathbf{x} ; \mathbf{x}_{s}\right)} \nabla^{2}\left[\int_{t} p_{s}\left(t, \mathbf{x} ; \mathbf{x}_{s}\right) p_{r}\left(t, \mathbf{x} ; \mathbf{x}_{r}\right) d t\right] .
$$

With the objective of further improving the quality and meaning of image amplitudes, Kiyashchenko et al. (2007) and Op't Root et al. (2012) derive the inverse scattering imaging condition (ISIC) for reverse-time migration (RTM). The latter authors demonstrated, using microlocal analysis, that this image condition is an asymptotic inverse of Born modeling. Therefore, ISIC provides image amplitudes that are proportional to reflection coefficients.

According to Op't Root et al. (2012), the ISIC is given in the frequency domain by

$$
I_{r}(\mathbf{x})=\frac{1}{2 \pi} \sum_{s} \int_{\omega} d \omega \frac{1}{(-i \omega) P_{s} \overline{P_{s}}}\left[\overline{P_{s}} P_{r}-\frac{c^{2}(\mathbf{x})}{\omega^{2}} \nabla \overline{P_{s}} \cdot \nabla P_{r}\right],
$$

where $P_{s}=P_{s}\left(\omega, \mathbf{x} ; \mathbf{x}_{s}\right)$ and $P_{r}=P_{r}\left(\omega, \mathbf{x} ; \mathbf{x}_{s}\right)$ are the Fourier transforms of the (downgoing) source and (upgoing) receiver wavefields for a source at $\mathbf{x}_{s}$, downward continued to the imaging point $\mathbf{x}$. Moreover, the bar over a symbol denotes the complex conjugate operation. To satisfy imaging condition 2, the reverse wavefield $\left(P_{r}\right)$ in time domain $\left(p_{r}\right)$ is backpropagated by

$$
\frac{1}{c^{2}(\mathbf{x})} \frac{\partial^{2} p_{r}(\mathbf{x}, t)}{\partial t^{2}}-\nabla^{2} p_{r}^{2}(\mathbf{x}, t)=F_{B} d\left(\mathbf{x}_{r}, t\right),
$$

where $d\left(\mathbf{x}_{r}, t\right)$ is the recorded data, injected at the position of the receivers and $F_{B}$ a boundary operator, defined as:

$$
F_{B}=-2 i D_{t} \frac{1}{c(\mathbf{x})} \sqrt{1-c^{2} D_{t}^{-2} D_{\mathbf{x}}^{2}}
$$

with

$$
D_{t}=-i \partial_{t}, \text { and } D_{\mathbf{x}}=-i \partial_{\mathbf{x}}
$$

Equation 2 is slightly different from the one of Op't Root et al. (2012). For simplicity, we have assumed that the source wavelet is a (possibly band-limited) delta-function, the effects of which are acceptable in the final migrated image. Therefore, we have combined in equation 2 the Green's function and source wavelet in the formula of Op't Root et al. (2012) into the source wavefield $P_{s}$. Moreover, we have made the sum over all sources explicit. Finally, the different sign of the factor $(-i \omega)$ in the denominator of the above equation results from our use of a different definition of the Fourier transform pair, being

$$
f(\omega)=\int_{-\infty}^{\infty} d t f(t) e^{i \omega t}, f(t)=\frac{1}{2 \pi} \int_{-\infty}^{\infty} d \omega f(\omega) e^{-i \omega t} .
$$

In this work, our objective is to find a time-domain version of equation 2 that can be efficiently implemented, and to study the image differences resuting from different implementational forms. For this purpose, we use a local high-frequency approximation to derive a number of theoretically equivalent time-domain forms and test their practical performance. In a similar way to Douma et al. (2010), we show that the ISIC for RTM can 
be reformulated into a version containing the Laplacian operator. Our theoretical analysis demonstrates that the Laplacian filter should be applied before illumination compensation. Moreover, we numerically evaluate the derived time-domain versions of the ISIC by comparing the resulting migrated images of synthetic data from the Marmousi II model, simulated using Born modeling.

\section{Implementational forms of ISIC}

The advantage of the frequency-domain representation of the ISIC in equation 2 is that the time derivatives are represented by factors $(-i \omega)$. Thus, it immediately allows us to recognize that these factors can be rather freely redistributed among the wavefield terms. Making use of this freedom, our first manipulation is to put the $\omega^{2}$ factor in the denominator of the spatial-derivatives term, where it would represent a two-fold time integration, in evidence. This results in

$$
\begin{aligned}
I_{r}(\mathbf{x})=\frac{1}{2 \pi} \sum_{s} \int_{\omega} d \omega \frac{1}{(-i \omega)^{3} P_{S} \overline{P_{S}}}[ & (-i \omega) \overline{P_{S}}(-i \omega) P_{r} \\
& \left.+c^{2}(\mathbf{x}) \nabla \overline{P_{S}} \cdot \nabla P_{r}\right],
\end{aligned}
$$

where we distributed the two $(-i \omega)$ factors inside the brackets symmetrically between the wavefield terms. This is the most conventional form of applying the time derivatives.

If we move one of the $(-i \omega)$ factors in the denominator to the receiver wavefield $P_{r}$, we can rewrite equation 7 as

$$
\begin{aligned}
I_{r}(\mathbf{x})=\frac{1}{2 \pi} \sum_{s} \int_{\omega} d \omega \frac{1}{(-i \omega)^{2} P_{s} \overline{P_{s}}}[ & (-i \omega) \overline{P_{s}}(-i \omega) Q_{r} \\
& \left.+c^{2}(\mathbf{x}) \nabla \overline{P_{s}} \cdot \nabla Q_{r}\right],
\end{aligned}
$$

where we have introduced the time-integrated receiver wavefield, given in the frequency domain by

$$
Q_{r}=Q_{r}\left(\omega, \mathbf{x} ; \mathbf{x}_{s}\right)=\frac{P_{r}\left(\omega, \mathbf{x} ; \mathbf{x}_{s}\right)}{(-i \omega)},
$$

or in the time domain by

$$
q_{r}\left(t, \mathbf{x} ; \mathbf{x}_{s}\right)=\int_{0}^{t} p_{r}\left(t^{\prime}, \mathbf{x} ; \mathbf{x}_{S}\right) d t^{\prime} .
$$

Under the assumption that the absolute value of the source wavefield is locally frequency independent, which is consistent with the local high-frequency approximation $P_{s}\left(\omega, \mathbf{x} ; \mathbf{x}_{s}\right)=A_{s}\left(\mathbf{x} ; \mathbf{x}_{s}\right) e^{-i \omega \tau_{s}\left(\mathbf{x} ; \mathbf{x}_{s}\right)}$, the illumination-compensation factor in the denominator can be taken out of the frequency integral (see also Kiyashchenko et al., 2007; Schleicher et al., 2008). In this way, equation 8 can be approximated by

$$
\begin{aligned}
I_{r}(\mathbf{x})=\frac{1}{2 \pi} \sum_{s} \frac{1}{P^{\prime}\left(\mathbf{x} ; \mathbf{x}_{s}\right)} \int_{\omega} d \omega & {\left[(-i \omega) \overline{P_{S}}(-i \omega) Q_{r}\right.} \\
& \left.+c^{2}(\mathbf{x}) \nabla \overline{P_{s}} \cdot \nabla Q_{r}\right],
\end{aligned}
$$

or in the time domain,

$$
\begin{array}{r}
I_{r}(\mathbf{x})=\sum_{s} \frac{1}{P^{\prime}\left(\mathbf{x}, \mathbf{x}_{s}\right)} \int_{t} d t\left[\dot{p}_{s}\left(t, \mathbf{x} ; \mathbf{x}_{s}\right) \dot{q}_{r}\left(t, \mathbf{x} ; \mathbf{x}_{s}\right)\right. \\
\left.+c^{2}(\mathbf{x}) \nabla p_{s}\left(t, \mathbf{x} ; \mathbf{x}_{s}\right) \cdot \nabla q_{r}\left(t, \mathbf{x} ; \mathbf{x}_{s}\right)\right],
\end{array}
$$

where the dot above a symbol denotes the time derivative. The illumination compensation factor $P^{\prime}\left(\mathbf{x}, \mathbf{x}_{s}\right)$ is given by the autocorrelation of the time derivative of the source wavefield. Equation 12 is the first implementational form of ISIC discussed in this paper. In the same way, we will keep using the local high-frequency approximation consistently for all remaining versions of the ISIC.

Another way of representing the ISIC in the time domain is to use the triple integral of the receiver's wavefield, which we denote by

$$
Q_{r}^{(3)}=Q_{r}^{(3)}\left(\omega, \mathbf{x} ; \mathbf{x}_{s}\right)=\frac{P_{r}}{(-i \omega)^{3}}=-\frac{Q_{r}}{\omega^{2}},
$$

or, in the time domain,

$$
q_{r}^{(3)}\left(t, \mathbf{x} ; \mathbf{x}_{s}\right)=\int_{0}^{t} q_{r}^{(2)}\left(t^{\prime}, \mathbf{x} ; \mathbf{x}_{s}\right) d t^{\prime} .
$$

With this notation, we can rewrite equation 8 as

$$
\begin{aligned}
I_{r}(\mathbf{x})=\frac{1}{2 \pi} \sum_{s} \frac{1}{P\left(\mathbf{x} ; \mathbf{x}_{S}\right)} \int_{\omega} d \omega[ & (-i \omega) \overline{P_{S}}(-i \omega) Q_{r}^{(3)} \\
& \left.+c^{2}(\mathbf{x}) \nabla \overline{P_{S}} \cdot \nabla Q_{r}^{(3)}\right],
\end{aligned}
$$

or in the time domain,

$$
\begin{aligned}
I_{r}(\mathbf{x})=\sum_{s} \frac{1}{P\left(\mathbf{x}, \mathbf{x}_{s}\right)} \int_{t} d t\left[\dot{p}_{s}\left(t, \mathbf{x} ; \mathbf{x}_{s}\right) \dot{q}_{r}^{(3)}\left(t, \mathbf{x} ; \mathbf{x}_{s}\right)\right. \\
\left.+c^{2}(\mathbf{x}) \nabla p_{s}\left(t, \mathbf{x} ; \mathbf{x}_{s}\right) \cdot \nabla q_{r}^{(3)}\left(t, \mathbf{x} ; \mathbf{x}_{S}\right)\right] .
\end{aligned}
$$

In this expression, the illumination factor $P\left(\mathbf{x} ; \mathbf{x}_{s}\right)$ is given by the autocorrelation of the source wavefield.

Another alternative form of the ISIC is obtained when using the second time derivative of the source wavefield given by

$$
r_{s}=\ddot{p}_{s} \quad \text { i.e., } \quad R_{s}=-\omega^{2} P_{s} .
$$

Adequate distribution of the $(-i \omega)$ factors in equation 8 leads to the expression

$$
\begin{aligned}
I_{r}(\mathbf{x})=\frac{1}{2 \pi} \sum_{s} \frac{1}{R\left(\mathbf{x} ; \mathbf{x}_{s}\right)} \int_{\omega} d \omega[ & (-i \omega) \overline{R_{s}}(-i \omega) Q_{r} \\
& \left.+c^{2}(\mathbf{x}) \nabla \overline{R_{s}} \cdot \nabla Q_{r}\right],
\end{aligned}
$$

or in the time domain,

$$
\begin{aligned}
I_{r}(\mathbf{x})=\sum_{s} \frac{1}{R\left(\mathbf{x}, \mathbf{x}_{S}\right)} \int_{t} d t\left[\dot{r}_{s}\left(t, \mathbf{x} ; \mathbf{x}_{S}\right) \dot{q}_{r}\left(t, \mathbf{x} ; \mathbf{x}_{S}\right)\right. \\
\left.+c^{2}(\mathbf{x}) \nabla r_{s}\left(t, \mathbf{x} ; \mathbf{x}_{s}\right) \cdot \nabla q_{r}\left(t, \mathbf{x} ; \mathbf{x}_{s}\right)\right] .
\end{aligned}
$$

In this version of the ISIC, the illumination compensation factor $R\left(\mathbf{x}, \mathbf{x}_{s}\right)$ is given by the autocorrelation of the second time derivative of the source wavefield.

\section{Relationship with the Laplacian}

It is not hard to conceive that the redistribution of the $(-i \omega)$ factors in equation 8 needs not be done symmetrically with respect to the involved wavefields. Instead of applying 
one factor to the source wavefield and the second one to the receiver wavefields, we can apply both factors to only one of these wavefields, say, the source wavefield. For convenience, we also put the squared velocity into evidence to obtain

$$
I_{r}(\mathbf{x})=\frac{1}{2 \pi} \sum_{s} \int_{\omega} d \omega \frac{c^{2}(\mathbf{x})}{P_{s} \overline{P_{s}}}\left[-\frac{\omega^{2}}{c^{2}(\mathbf{x})} \overline{P_{s}} Q_{r}^{(3)}+\nabla \overline{P_{s}} \cdot \nabla Q_{r}^{(3)}\right] .
$$

Below, we show that there are different possible ways to interpret this formula.

\section{Second-order source-wavefield derivative}

On the one hand, the factor $\omega^{2} / c^{2}$ in equation 20 can be interpreted as multiplying the source wavefield. Upon the use of the corresponding Helmholtz equation,

$$
\nabla^{2} P_{s}+\frac{\omega^{2}}{c^{2}(\mathbf{x})} P_{s}=0
$$

equation 20 becomes

$$
\begin{aligned}
I_{r}(\mathbf{x}) & =\frac{1}{2 \pi} \sum_{s} \int_{\omega} d \omega \frac{c^{2}(\mathbf{x})}{P_{S} \overline{P_{s}}}\left[\nabla^{2} \overline{P_{s}} Q_{r}^{(3)}+\nabla \overline{P_{s}} \cdot \nabla Q_{r}^{(3)}\right] \\
& =\frac{1}{2 \pi} \sum_{s} \frac{c^{2}(\mathbf{x})}{P\left(\mathbf{x} ; \mathbf{x}_{s}\right)} \int_{\omega} d \omega \nabla \cdot\left[\nabla \overline{P_{s}} Q_{r}^{(3)}\right],
\end{aligned}
$$

where the second equality is a result of applying the local high-frequency approximation and recognizing the product rule for the spatial derivatives.

\section{Second-order receiver-wavefield derivative}

On the other hand, we can also interpret the factor $\omega^{2} / c^{2}$ in equation 20 as multiplying the receiver wavefield. Using the (third time integral of the) associated Helmholtz equation,

$$
\nabla^{2} Q_{r}^{(3)}+\frac{\omega^{2}}{c^{2}(\mathbf{x})} Q_{r}^{(3)}=0
$$

we can rewrite equation 20 as

$$
I_{r}(\mathbf{x})=\frac{1}{2 \pi} \sum_{s} \frac{c^{2}(\mathbf{x})}{P\left(\mathbf{x} ; \mathbf{x}_{s}\right)} \int_{\omega} d \omega \nabla \cdot\left[\overline{P_{s}} \nabla Q_{r}^{(3)}\right] .
$$

\section{Laplacian filter}

Moreover, since equations 22 and 24 represent manipulations of the same theoretical expression 8 , we can also calculate the image $I_{r}$ as the average of both expressions, i.e.,

$$
\begin{aligned}
I_{r}(\mathbf{x}) & =\frac{1}{2 \pi} \sum_{s} \frac{c^{2}(\mathbf{x})}{P\left(\mathbf{x} ; \mathbf{x}_{s}\right)} \frac{1}{2} \int_{\omega} d \omega\left(\nabla \cdot\left[\nabla \overline{P_{s}} Q_{r}^{(3)}\right]+\nabla \cdot\left[\overline{P_{s}} \nabla Q_{r}^{(3)}\right]\right) \\
& =\frac{1}{2 \pi} \sum_{s} \frac{c^{2}(\mathbf{x})}{2 P\left(\mathbf{x} ; \mathbf{x}_{s}\right)} \nabla^{2} \int_{\omega} d \omega\left[\overline{P_{s}} Q_{r}^{(3)}\right]
\end{aligned}
$$

where we again have made use of the product rule for the gradient. Finally, in the time domain, this equation reads

$$
I_{r}(\mathbf{x})=\sum_{s} \frac{c^{2}(\mathbf{x})}{2 P\left(\mathbf{x} ; \mathbf{x}_{S}\right)} \nabla^{2} \int_{t} d t\left[p_{s}\left(t, \mathbf{x} ; \mathbf{x}_{S}\right) q_{r}^{(3)}\left(t, \mathbf{x} ; \mathbf{x}_{S}\right)\right] .
$$

We note that, except for a small modification, the ISIC in this form is equivalent to applying the Laplacian to the correlation of the source and receiver wavefields, which is a common procedure in practice. The theoretical derivation demonstrates that the Laplacian must be applied before the illumination compensation. Moreover, there is a minor amplitude modification, represented by the multiplication with a factor of $c^{2} / 2$. Finally, to get the wavelet shape correct, version 26 makes use of the third time integral of the receiver wavefield.

Of course, the same manipulations can be applied to the form 18 of the ISIC, resulting in

$$
I_{r}(\mathbf{x})=\frac{1}{2 \pi} \sum_{s} \frac{c^{2}(\mathbf{x})}{2 R\left(\mathbf{x} ; \mathbf{x}_{S}\right)} \nabla^{2} \int_{\omega} d \omega\left[\overline{R_{S}} Q_{r}\right],
$$

or in the time domain,

$$
I_{r}(\mathbf{x})=\sum_{s} \frac{c^{2}(\mathbf{x})}{2 R\left(\mathbf{x} ; \mathbf{x}_{s}\right)} \nabla^{2} \int_{t} d t\left[r_{s}\left(t, \mathbf{x} ; \mathbf{x}_{s}\right) q_{r}\left(t, \mathbf{x} ; \mathbf{x}_{s}\right)\right] .
$$

In this version, the wavelet shape is corrected by using the second derivative of the source wavefield together with the integrated receiver wavefield. The application of the Laplacian before illumination compensation and the amplitude correction by a factor $c^{2} / 2$ remain the same as in equation 26.

\section{Numerical Examples}

To evaluate the performance of the above-discussed versions of the ISIC, we have implemented and applied them in a reverse-time migration of synthetic Born data from the Marmousi II model. All migrations use the same algorithm for the wavefields propagation in a smoothed version of the Marmousi II model (Figure 1). In Figure 2, we show the true reflectivity model (Figure 2a) and the RTM images resulting from applying the different ISIC versions. Figure $2 \mathrm{~b}$ shows the RTM image using Claerbout's original crosscorrelation imaging condition with Laplacian filter and illumination compensation. Correspondingly, Figures 2c, $2 \mathrm{~d}, 2 \mathrm{e}, 2 \mathrm{f}$, and $2 \mathrm{~g}$ show the RTM images obtained using the implementational forms of the ISIC in time domain as discussed above, equations 12, 16, 19, 26, and 28, respectively.

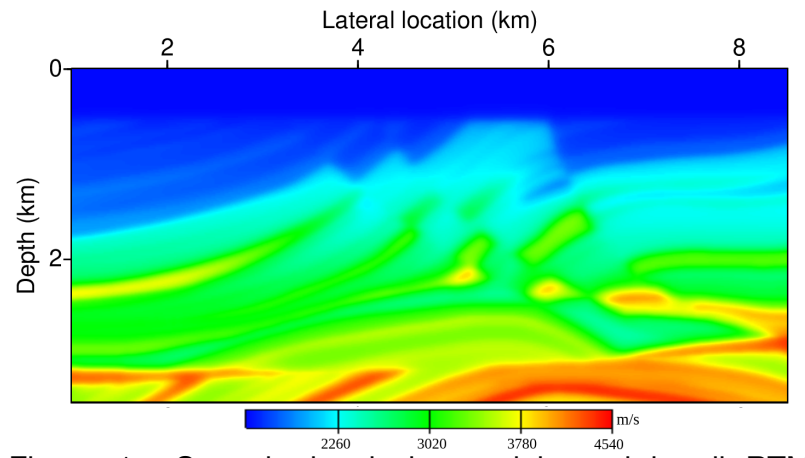

Figure 1: Smoothed velocity model used in all RTM images.

Comparing the RTM image obtained using Claerbout's image condition (Figure $2 \mathrm{~b}$ ) with the true reflectivity model convolved with the same source pulse used in the RTM images (Figure 2a), we note that this imaging condition, 


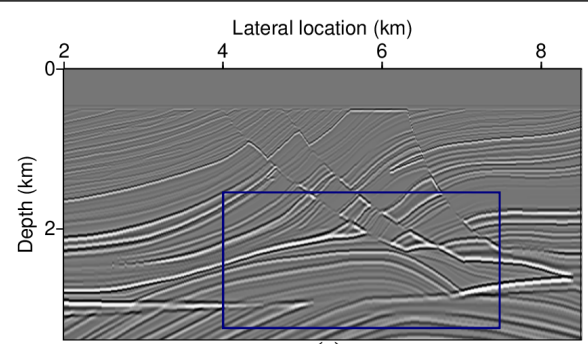

(a)



(b)

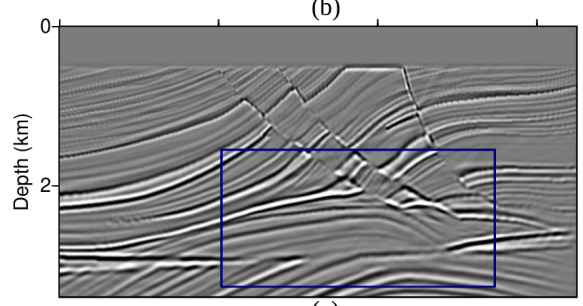

(c)

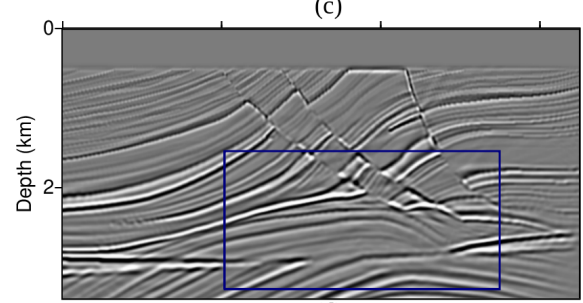

(d)

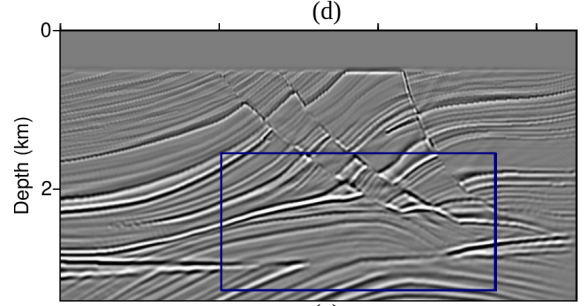

(e)

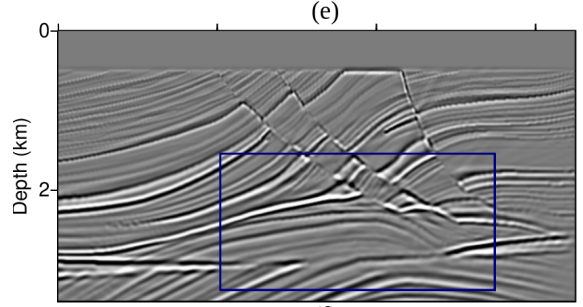

(f)

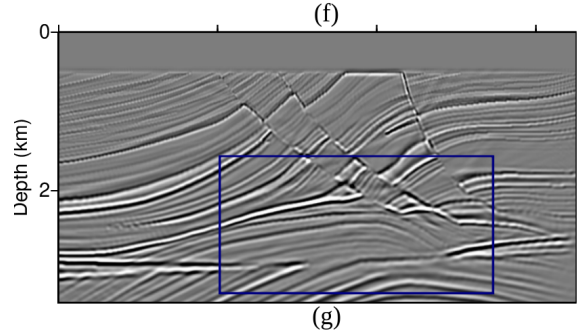

(g)

Figure 2: RTM images. (a) True reflectivity model convolved with source pulse; (b) RTM image using Claerbout's imaging condition with Laplacian filter and amplitude compensation 1; (c-g) RTM images using the respective implementational forms of ISIC: (c) equation 12, (d) equation 16, (e) equation 19, (f) equation 26, and (g) equation 28. although detecting a large part of the model's structures, distorts the images' amplitudes, so that they are no longer proportional to the reflection coefficients of the true model.

In contrast, all RTM images obtained using the different implementational forms of the ISIC present much better amplitudes. At first look, the five images shown in Figures $2 \mathrm{c}, 2 \mathrm{~d}, 2 \mathrm{e}, 2 \mathrm{f}$, and $2 \mathrm{~g}$ look rather similar, reflecting the theoretical equivalence between the applied imaging conditions. Comparing them visually with the true reflectivity model convolved with source pulse of Figure $2 \mathrm{a}$, we note that the amplitudes generally provide a good approximation of the true reflectivity.

For a closer inspection, we show in Figure 3 a detail of the ISIC images and the reflectivity model (region delimited by the blue rectangle in Figure 2). Again, the overall impression of rather similar images persists, and all images compare favorably with the reflectivity model (Figure 3a). However, in the zoom, we can now recognize a few subtle differences. Generally speaking, the images of Figures 3b, $3 \mathrm{c}$, and $3 \mathrm{e}$, which don't use the second time-derivative of the source wavefield (see equations 12, 16, and 19), are identical and appear to present a slightly inferior resolution to the other two images, Figures $3 d$ and $3 f$, which were obtained using the ISIC versions employing the second time-derivative of the source wavefield (equations 26 and 28).

An evaluation of the achieved recovery of the reflection coefficients can be better achieved by a trace-by-trace comparison than by looking at the complete images. Figure 4 shows such a comparison of a single trace of the true model reflectivity, taken at the horizontal position of $4500 \mathrm{~m}$, to the corresponding traces of the RTM images. All traces have been normalized to their maximum amplitudes.

We immediately note that the ISIC traces (Figures 4c, 4d, $4 \mathrm{e}, 4 \mathrm{f}$, and $4 \mathrm{~g}$ ) present a similar amplitudes distribution, doing a much better job of representing the true reflectivity than the trace in Figure $4 \mathrm{~b}$, obtained using Claerbout's imaging condition with illumination compensation and Laplacian filtering (equation 1). This difference is more significant in the first layers of the image (indicated by blue background color) where, unlike the true trace (Figure 4a), the amplitudes of Claerbout's imaging condition start with higher values and detect the structure in about 1300 meters with less amplitude (Figure 4b). Also, in the deepest part of the traces (purple background), amplitudes of structures below 3000 meters are very low. These effects are corrected in all traces produced with the different versions of the ISIC (Figures 4c, 4d, 4e, 4f, and 4g). We note that the traces obtained using second time-derivative (Figures $4 \mathrm{e}$ and $4 \mathrm{~g}$ ) present the best resolution and, thus, the highest similarity to the reflectivity trace.

\section{Computational cost}

We tested all imaging conditions using the same routines for the wavefield propagation, with a code parallelized using GPUs with 38 threads per processor. We measured the time considering only the heaviest stages of computing: numerical evolution of the forward and backward wavefields and application of the imaging condition. Table 1 shows the computational cost for migration using each imaging condition discussed in this paper. Note the the Laplacian versions of ISIC (equations 26 and 28) have the same 


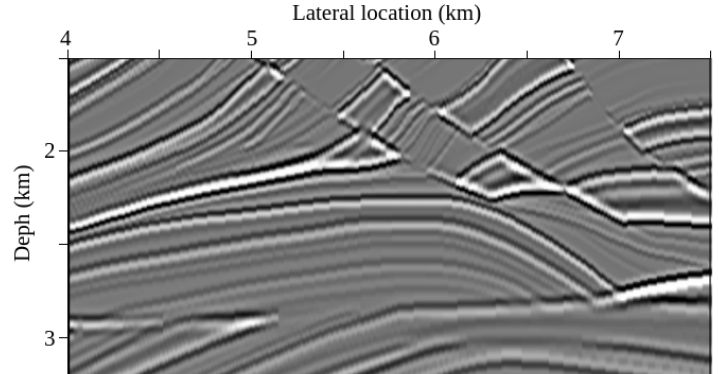

(a)



(c)

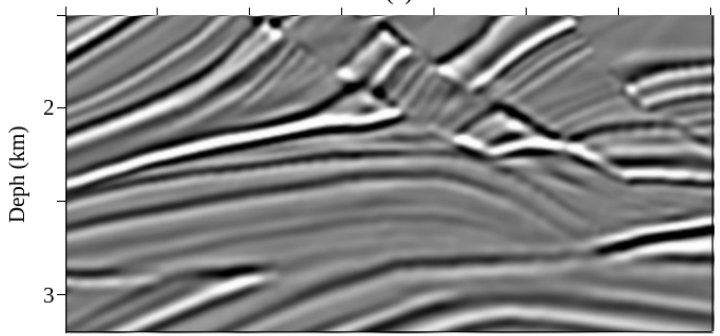

(e)



(b)

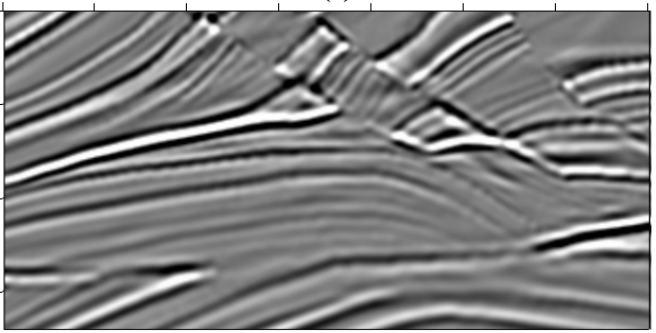

(d)

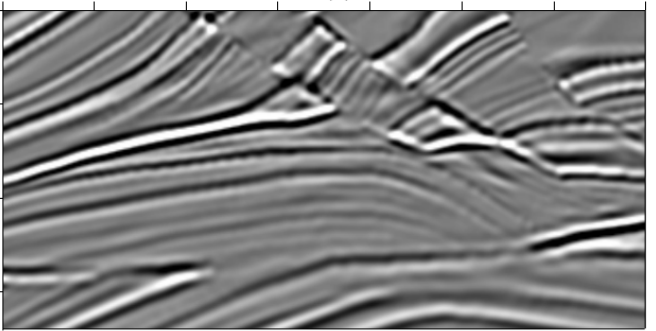

(f)

Figure 3: Detail of the reflectivity model and ISIC images (region delimited by the blue rectangle in Figure 2). (a) True reflectivity model convolved with source pulse; (b-f) RTM images using the ISIC implementational forms: (b) equation 12, (c) equation 16, (d) equation 19, (e) equation 26, and (f) equation 28.

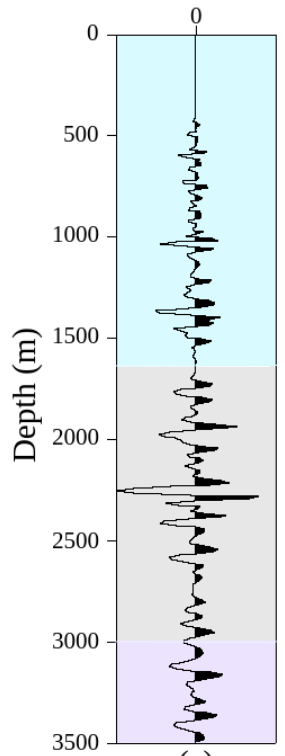

(a)

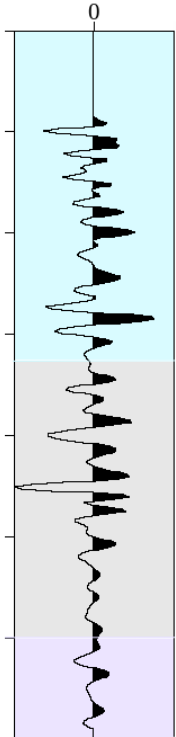

(b)

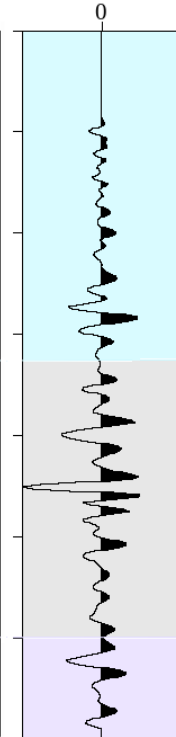

(c)

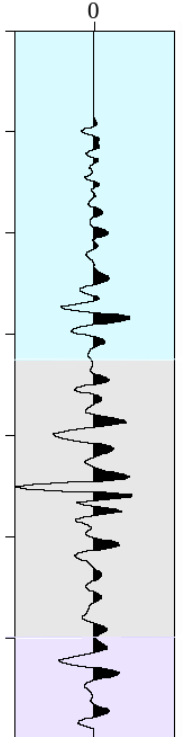

(d)

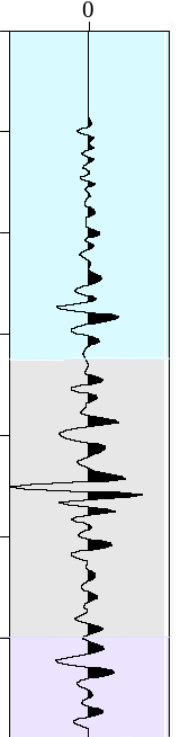

(e)

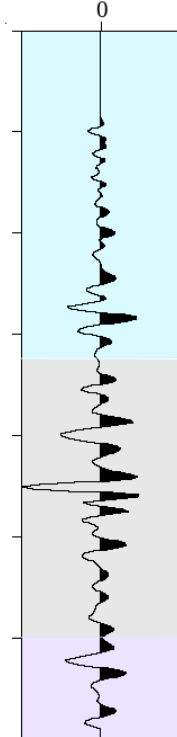

(f)

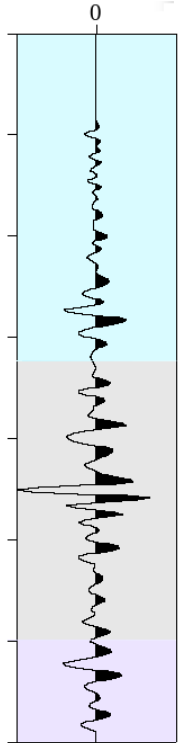

(g)

Figure 4: Trace of reflectivity and RTM images at 4500 meters. (a) True reflectivity model convolved with source pulse; (b) RTM image using Claerbout's imaging condition with Laplacian filter and amplitude compensation, equation 1; (c-g) RTM images using the respective implementational forms of ISIC: (c) equation 12, (d) equation 16, (e) equation 19, (f) equation 26, and (g) equation 28. 
computational cost as Claerbout's imaging condition with Laplacian filter and illumination compensation (equation 1), but result in better image quality.

Table 1: Computational cost of each imaging condition

\begin{tabular}{r|cc} 
& Equation & time $(\mathrm{min})$ \\
\hline Claerbout's IC-LI & 1 & 43.0 \\
\hline & 12 & 51.0 \\
ISIC & 16 & 50.5 \\
& 19 & 50.2 \\
& 26 & 43.0 \\
& 28 & 42.9
\end{tabular}

Implementational peculiarities of ISIC using the third integral of the receiver's wavefield

The third integral used in equations 16 and 26 causes an imbalance of the low frequencies in the migrated image. To avoid this problem, before injecting the third integral of the data in the receiver's position, it is necessary to apply a filter to regulate the low frequencies present in the data. In this work, we make use of a leaky integration (Claerbout, 2014), i.e,

$$
y(t)=\int_{0}^{\infty} x(t-\tau) \rho d \tau,
$$

where $\rho=e^{-\alpha \tau}$. Throughout integration, $\rho$ is a constant that can admit a value from 0 to 1 . In our numeric experiments, we choose $\rho=0.99$. In the case of triple integration, Equation 29 is recursively applied in the seismic data three times.

\section{Conclusions}

In this paper, we have deduced and implemented a number of theoretically equivalent versions of the trueamplitude inverse-scattering imaging condition for RTM of Kiyashchenko et al. (2007) and Op't Root et al. (2012). In a similar way to Douma et al. (2010), our theoretical derivations have show a relationship between the trueamplitude imaging condition for RTM and the Laplacian operator. This operator is frequently used in seismic imaging without a profound theoretical basis to remove low-frequency backscattering artefacts from RTM images. The derivations have demonstrated that the Laplacian filter needs to be applied before the illumination compensation.

Through numerical tests using Born data from Marmousi II, we have shown that the ISIC in all cases provides results superior to a simple cross-correlation of the source and receiver wavefields, even with the application of Laplacian filter and illumination compensation. We have seen that the ISIC implementational forms studied in this paper, though being theoretically equivalent, produce numerically similar but not identical results.

In our numerical experiments for ISIC, formulations based on the Laplacian and with two cross-correlation terms that consider the second time derivative of the source wavefield show a slight improvement in resolution over the other formulations. The conventional form of ISIC presents a better result than simple cross-correlation but has a higher computational cost. We also showed that the proposed forms of ISIC based on the Laplacian provide very similar results to the conventional form of ISIC, but at the same cost as the imaging condition of Claerbout (1971) with Laplacian filter and illumination compensation. Thus, we conclude that the best form of the ISIC is the Laplacian version using the second time-derivative of the source wavefield.

\section{Acknowledgements}

This work was kindly supported by Petrobras, the Brazilian agencies CNPq and CAPES, and the sponsors of the Wave Inversion Technology (WIT) Consortium.

\section{References}

Baysal, E., D. Kosloff, and W. Sherwood, 1983, Reverse time migration: Geophysics, 48, 1514-1524.

Biondi, B. L., 2006, 3D seismic imaging: Society of Exploration Geophysicists.

Claerbout, J. F., 1971, Toward a unified theory of reflector mapping: Geophysics, 36, 467-481.

- 2014, Geophysical image estimation by example: Lulu. com.

Douma, H., D. Yingst, I. Vasconcelos, and J. Tromp, 2010, On the connection between artifact filtering in reversetime migration and adjoint tomography: GEOPHYSICS, 75, S219-S223.

Kiyashchenko, D., R.-E. Plessix, B. Kashtan, and V. Troyan, 2007, A modified imaging principle for true-amplitude wave-equation migration: Geophysical Journal International, 168, 1093-1104.

McMechan, G. A., 1983, Migration by extrapolation of timedependent boundary values: Geophysical Prospecting, 31, 413-420.

Op't Root, T. J., C. C. Stolk, and V. Maarten, 2012, Linearized inverse scattering based on seismic reverse time migration: Journal de mathématiques pures et appliquées, 98, 211-238.

Schleicher, J., J. C. Costa, and A. Novais, 2008, Timemigration velocity analysis by image-wave propagation of common-image gathers: Geophysics, 73, VE161VE171.

Schultz, P. S., and J. W. C. Sherwood, 1980, Depth migration before stack: Geophysics, 45, 376-393. 